\title{
A RESISTÊNCIA ANTIMICROBIANA E CUSTOS DE CUIDADO DE SAÚDE: UMA REVISÃO SISTEMÁTICA
}

Tiago de Oliveira Silva, Luis do Nascimento Ortega

Universidade do Oeste Paulista - UNOESTE, Presidente Prudente, SP. E-mail: tiagosilva46@outlook.com

\section{RESUMO}

Observa-se uma crescente preocupação acerca das capacidades multirresistentes apresentadas pelos microrganismos aos antimicrobianos. A sociedade é prejudicada com o redirecionamento de recursos financeiros de outras áreas, também carentes à população, para a compra de antimicrobianos mais potentes e em maiores quantidades. O presente estudo descreve o consumo de fármacos antimicrobianos e seu impacto gerado por meio de uma revisão sistemática, seguindo as recomendações do método Prisma. Foram realizadas buscas em quatro bases de dados (Portal BVS, PubMed, Embase e Science Direct). Foram encontrados 196 artigos, sendo selecionados 11 após aplicação dos critérios de elegibilidade. Um estudo prospectivo demonstrou que a diferença nos gastos pode chegar a $\$ 31,17$ doentes/dias entre profiláticos e com infecções nosocomiais. Os estudos mostraram que o acréscimo de custos está relacionado com o aumento no período de internação. Mortalidade e internação em UTI também se elevaram. Recomenda-se a realização de mais estudos com níveis de evidência elevados.

Palavras-chave: resistência antimicrobiana, impacto financeiro, finanças públicas, fármacos antimicrobianos, assistência à saúde.

\section{ANTIMICROBIAL RESISTANCE AND HEALTH CARE COSTS: A SYSTEMATIC REVIEW}

\section{ABSTRACT}

There is a growing concern about the multi-resistant capabilities presented by microorganisms to antimicrobials. Society is harmed by the redirection of financial resources from other areas, also lacking the population, to purchase more potent antimicrobials and in larger quantities. This study describes the consumption of antimicrobial drugs and their impact generated through a systematic review, following the recommendations of the Prisma method. Searches were performed in four databases (Portal BVS, PubMed, Embase and Science Direct). A total of 196 articles were found, 11 of which were selected after applying the eligibility criteria. A prospective study showed that the difference in expenditures can reach $\$ 31.17$ patients/day between prophylactics and those with nosocomial infections. Studies have shown that the increase in costs is related to the increase in the length of stay. Mortality and admission to the ICU also increased. Further studies with high levels of evidence are recommended.

Keywords: antimicrobial resistance, financial impact, public finances, antimicrobial drugs, health care.

\section{INTRODUÇÃO}

O advento do fármaco antimicrobiano é considerado um dos maiores avanços da medicina moderna. Enfermidades como a meningite, pneumonia, tuberculose, sífilis, dentre outras doenças infecciosas, que na era pré- antibiótica eram tidas como verdadeiras sentenças de morte, passaram a ser tratáveis ${ }^{1}$.

Antimicrobianos são compostos químicos obtidos por meio do metabolismo secundário de microrganismos (antibióticos) ou de maneira sintética (quimioterápicos), são capazes de destruir ou inibir o crescimento dos 
microrganismos patogênicos, sem trazer grandes consequências ao hospedeiro graças à sua toxidade seletiva ${ }^{1,2}$.

São classificados principalmente quanto à sua ação biológica (bactericidas, quando capazes de eliminar microrganismos, ou bacteriostáticos, quando inibem a sua reprodução), seu espectro de ação, determinado pela eficiência contra determinado tipo de micróbio (ação principal contra bactéria Gram-negativa, Gram-positiva, anaeróbias, espiroquetas, fungos, entre outros) e mecanismo de ação (podendo atuar inibindo sínteses da parede celular, proteica e de ácidos nucleicos, ou alterando a permeabilidade da membrana plasmática do microrganismo) $)^{1,3}$.

O otimismo provocado pela difusão do uso clínico da penicilina em meados da década de 1940 foi logo extinto pelo aparecimento da Staphylococcus aureus resistente capaz de sintetizar a enzima penicilinase. Antes de 1946, cerca de $90 \%$ dos isolados de S. aureus em hospitais eram suscetíveis à penicilina, já em 1952 este número havia reduzido para apenas $25 \%, 4,6$.

A Organização Mundial da Saúde (OMS) define resistência antimicrobiana (RAM) como a "capacidade de um microrganismo (como bactérias, vírus e alguns parasitas) de impedir que um antimicrobiano (como antibióticos, antivirais e antimaláricos) atue contra ele" ${ }^{\prime 7}$.

Os microrganismos fazem uso de diversas estratégias para resistir ao antimicrobiano por meio do desenvolvimento de mutações no gene, sendo capazes de alterar a permeabilidade da membrana do microrganismo, o sitio de ação do fármaco, as importantes vias metabólicas, além de estimular a síntese de enzimas e bombas de efluxo. As bactérias podem também adquirir estes mecanismos de resistência de maneira externa, através da transferência horizontal de genes (transformação, transdução e conjugação) ${ }^{3,}$ 8

Essa capacidade natural que o microrganismo possui de desenvolver a resistência antimicrobiana somada ao uso exacerbado e indiscriminado destes fármacos que atuam como selecionadores de cepas resistentes, tem levado ao surgimento de microrganismos multirresistentes por todo o mundo. Um estudo realizado pelo economista britânico Jim O'Neill aponta que anualmente, mais de 700 mil pessoas vão a óbito devido à resistência antimicrobiana, e caso este cenário não sofra alterações, até 2050 a RAM se tornará mais letal que o câncer, podendo atingir a marca de 10 milhões de mortes por ano ${ }^{9,}$ 10

A Organização Mundial da Saúde demonstra preocupação, tendo elaborado em 2015 um plano de ação global contra a resistência antimicrobiana, além de citar a RAM na lista das dez ameaças a saúde global em $2019^{11,12}$

Apesar da crescente demanda por antimicrobianos cada vez mais potentes, é incerto que novos fármacos cheguem ao mercado em um futuro próximo. O avanço da resistência, somada à necessidade de utilizar esses medicamentos com prudência tem desestimulado sua pesquisa e desenvolvimento nas indústrias farmacêuticas devido à baixa lucratividade ${ }^{13}$.

Além de provocar impactos na saúde, a RAM também impõe grandes perdas financeiras a sociedade. Um relatório publicado pela Organização para Cooperação e Desenvolvimento Econômico (OCDE) em 2018 estima que entre os anos de 2015 e 2050, o ônus gerado aos serviços de saúde dos 33 países incluídos no estudo poderá chegar a 3,5 bilhões de dólares por ano, corrigidos pela paridade do poder de compra (PPC), em decorrência de complicações ocasionadas por microrganismos resistentes ${ }^{14}$.

Tem se observado um aumento expressivo das capacidades multirresistentes dos microrganismos. O que não apenas provoca danos à saúde da comunidade de maneira geral, como também gera prejuízos as demais áreas de interesse da população como segurança, educação e infraestrutura, levando-se em conta os recursos que poderiam ser destinados a estas áreas sendo redirecionados para a compra de antimicrobianos cada vez mais potentes e em maior quantidade.

Diante do exposto, fica evidente a validade de se iniciar uma nova reflexão do ponto de vista econômico partindo se de uma revisão sistemática de publicações referentes ao assunto em questão, buscando auxiliar gestores e profissionais da saúde a optarem pela tomada de decisões mais adequada, garantindo o uso racional dos fármacos antimicrobianos e reduzindo seu impacto financeiro.

O presente estudo tem por objetivo descrever o custo de cuidados de saúde provocados pela resistência antimicrobiana, visando verificar os gastos, descrever o consumo e determinar os antimicrobianos mais consumidos. 


\section{MÉTODOS}

O presente estudo é uma revisão sistemática e busca seguir as recomendações do método Prisma ${ }^{15}$. Foi realizado um levantamento bibliográfico de produções indexadas nas seguintes bases de dados eletrônicas: Portal BVS, PubMed, Embase e Science Direct. Para a busca foram utilizadas palavras incluídas nos recursos dos Descritores em Ciências da Saúde (DeCS) e no Medical Subject Headings (MeSH), além de termos livres não encontrados nessas ferramentas. Foram incluídos estudos publicados no período de 2015 a 2020, nos idiomas: Inglês, português e espanhol.

A elaboração da pergunta que compõe o presente estudo utilizou o modelo Population, Intervention, Comparison, Outcome (PICO) como referência ${ }^{16}$. Onde ' $P$ ' se refere a população que faz o uso de medicamentos antimicrobianos, ' $\mathrm{l}$ ' a utilização inadequada do antimicrobiano, ' $C$ ' a comparação dessa população com pacientes que não apresentam resistência antimicrobiana e ' $O$ ' ao desfecho do estudo, com o aumento ou não dos custos à saúde.

As estratégias de busca para o portal da Biblioteca Virtual de Saúde (BVS) foram: ("resistência microbiana a medicamentos" OR "drug resistance, microbial" OR "farmacorresistencia microbiana" OR "resistência microbiana a antibióticos" OR "resistência microbiana a drogas" OR "resistência a antibióticos") AND ("custos de cuidados de saúde" OR "health care costs" OR "costos de la atención en salud" OR "custos de cuidados médicos" OR "custos de tratamento" OR "gastos em saúde") AND ( db:("MEDLINE" OR "LILACS") AND la:("en" OR "es" OR "pt")); para o PubMed foram: (drug resistance, microbial[MeSH Terms]) AND (Health Care Costs[MeSH Terms]); para o Science Direct foram: ("health care cost" OR "finance impact") AND ("resistance microbial" OR "drug resistance" OR "antimicrobial resistance"); e para a Embase ('antibiotic resistance'/exp/mj AND 'health care cost'/exp/mj.).
Foi utilizado o software Mendeley Desktop na versão 1.19.4 para indexar os trabalhos selecionados.

Para a seleção dos artigos foram incluídos estudos primários. Inicialmente foram excluídos aqueles estudos que apresentaram duplicidade, além de demais artigos de revisão. Sendo selecionados após leitura na seguinte ordem: do título, resumo e do artigo em si na sua integralidade.

Para facilitar na extração e organização dos dados foi desenvolvido um formulário padronizado que contém informações quanto ao nome do artigo, nome dos autores, ano de publicação, tipo de estudo, país onde o estudo foi realizado, tamanho da amostra, desfechos, perfil de resistência microbiológica e custos hospitalares totais relacionados à resistência antimicrobiana.

\section{RESULTADOS}

Após a finalização da etapa de buscas com base nas estratégias previamente estabelecidas, foram localizados ao todo 196 estudos, sendo 137 na base de dados BVS, 29 na Embase, 25 na Pubmed e 5 na Sciense Direct. Ao seguir os critérios de seleção 5 estudos duplicados foram removidos. Outros 161 artigos foram desconsiderados após leitura do título e resumo por não apresentarem relação com o objetivo dessa revisão. Por fim, após leitura completa dos 30 artigos restantes, optou-se por não incluir 19 estudos, sendo o principal fator determinante para a exclusão nessa etapa a inexistência de grupos controle. Após todas as etapas de seleção e aplicação dos critérios de elegibilidade, foram incluídos 11 estudos (Tabela 2): 7 de coorte, sendo destes um do tipo prospectivo, e 4 estudos observacionais retrospectivos. 


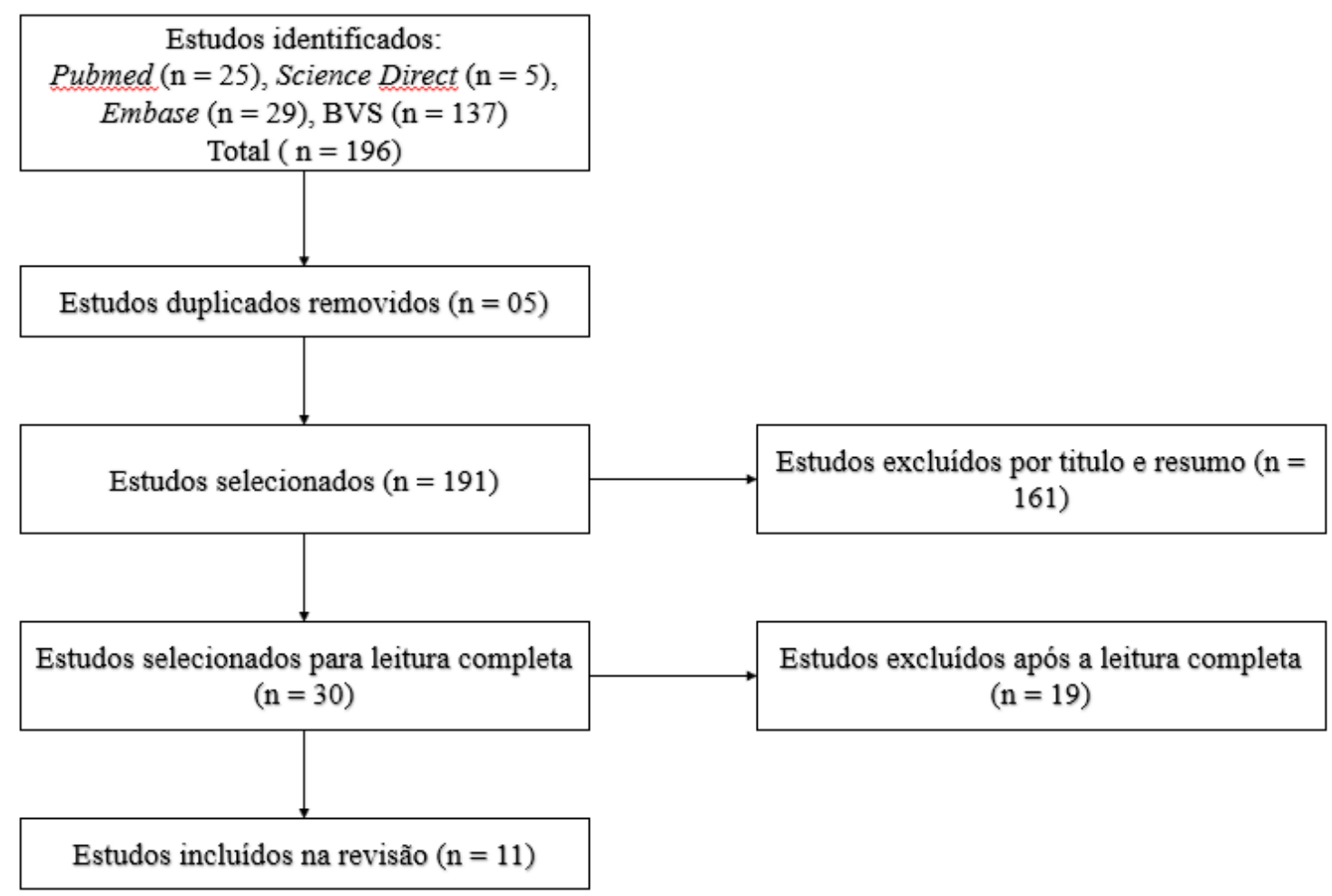

Figura 1. Fluxograma baseado no modelo Prisma ilustrando o processo de seleção dos estudos, adaptado pelo autor.

A Tabela 1 traz o número de estudos selecionados, agrupados pelo tipo de abordagem seguida quanto aos desfechos apresentados pelos mesmos.

Tabela 1. Tipo de abordagem, com número e porcentagem.

\begin{tabular}{lll}
\hline Tipo de abordagem & N & $\%$ \\
\hline Custos financeiros & 2 & 18,2 \\
\hline Custos financeiros e percentual de mortalidade & 1 & 9,1 \\
\hline Custos financeiros e tempo médio de internação & 2 & 18,2 \\
\hline Custos financeiros, tempo médio de internação e percentual de mortalidade & 4 & 36,3 \\
\hline $\begin{array}{l}\text { Custos financeiros, tempo médio de internação, percentual de mortalidade } \\
\text { e internação em leito de UTI }\end{array}$ & 2 & 18,2 \\
\hline
\end{tabular}

Para facilitar na visualização, os dados relevantes extraídos dos onze estudos selecionados foram alocados na Tabela 3, que agrupa variáveis como: nome dos autores, tipo de estudo, tamanho da amostra, principais antimicrobianos e ônus provocado pela resistência aos antimicrobianos (financeiro, mortalidade, período médio de internação e necessidade de atendimento em UTI). Todos os valores monetários cuja moeda não era o dólar foram convertidos para dólares americanos de acordo com o ano de publicação referente ao estudo, a fim de se obter uma melhor padronização.
Cinco artigos detalharam o perfil de resistência microbiológico encontrado em suas populações de estudo. O estudo observacional retrospectivo feito em 2019 por Johnston KJ e colaboradores $^{21}$ nos Estados Unidos especifica que 312 mil das mais de 6 milhões de internações estudadas eram motivadas pela bactéria Clostridium difficile, já bactérias multirresistentes estavam presentes em 16 mil internações.

Outro estudo observacional retrospectivo também realizado nos Estados Unidos em 2019 por Nguyenl $\mathrm{HQ}$ e colaboradores ${ }^{22}$ analisando 546 mil internações detalhou que $4,45 \%$ $(n=24.358)$ das internações continham algum 
microrganismo resistente, $1,21 \% \quad(n=6.630)$ multirresistentes, $1,77 \%(n=9.712)$ de $S$. aureus resistente a meticilina (MRSA) e 0,49\% ( $n=2.708)$ de microrganismos resistentes a beta-lactâmicos (BR).

Um estudo de caso-controle realizado na Grécia por Kousouli E. e colaboradores ${ }^{23}$ em 2019 com 419 pacientes (142 casos/277 controles) apontou que cerca de $26 \%(n=37)$ destes eram acometidos pela $K$. pneumoniae resistente a carbapenem (CRKP), $35 \% \quad(n=50)$ pela $A$. baumannii resistente a carbapenem (CRAB), 8,4\% $(n=12)$ pela $P$. aeruginosa resistente a carbapemem (CRPA), 25,3\% por CRKP + CRAB $(n=36), 0,7 \%$ por CRKP + CRAB + CRPA: $0,7 \%$ $(n=1), 1,4 \%$ por CRKP + CRPA $(n=2)$ e $2,8 \%$ por CRAB + CRPA ( $n=4)$.

O estudo de coorte prospectivo feito por Axente $\mathrm{C}$ e colaboradores ${ }^{24}$ na Romênia em 2017 possuía o seguinte perfil microbiológico: Klebsiella pneumoniae produtora de $\beta$-lactamase de amplo espectro (ESBL): 55,91\% ( $n=123 / 220)$, Acinetobacter baumannii multidroga resistente (MDR): $54,47 \% \quad(n=73 / 134)$, Proteus mirabilis produtora de $\beta$-lactamase de amplo espectro (ESBL): 47,79\% ( $n=65 / 136)$, Estafilococo de coagulase positiva resistente à meticilina (MRCNS): 62,35\% ( $n=53 / 85), \quad$ Pseudomonas aeruginosa multidroga resistente (MDR): $37,93 \%$ $(n=52 / 137)$, Escherichia coli produtora de $\beta$ lactamase de amplo espectro (ESBL): 16,67\% ( $n=23 / 138$ ) e Enterobacter cloacae produtora de $\beta$-lactamase de amplo espectro (ESBL): $29,41 \%$ $(n=5 / 17)$.

Já o estudo de coorte retrospectivo observacional feito nos Estados Unidos em 2016 por Judd WR e colaboradores ${ }^{27}$ com 382 pacientes aponta que $8,7 \%(n=32)$ dos pacientes era acometida por Pseudomonas aeruginosa resistente a meropenem. 
Tabela 2. Estudos selecionados, com autores, nome do artigo, ano de publicação, periódico e país onde estudo foi realizado.

\begin{tabular}{|c|c|c|c|c|}
\hline Autores & Nome do artigo & Ano & Periódico & País \\
\hline $\begin{array}{l}\text { Phodha T, Riewpaiboon A, } \\
\text { Malathum A, Coyte } \text { PC }^{18}\end{array}$ & $\begin{array}{l}\text { Excess annual economic burdens from nosocomial infections } \\
\text { caused by multi-drug resistant bactéria in Thailand }\end{array}$ & 2019 & $\begin{array}{l}\text { Expert Review } \\
\text { Pharmacoeconomics } \\
\text { Outcomes Research }\end{array}$ & Tailândia \\
\hline $\begin{array}{l}\text { Zhen } \mathrm{X} \text {, Chen } \mathrm{Y}, \mathrm{Hu} \mathrm{X} \text {, Dong } \mathrm{P}, \mathrm{Gu} \\
\text { S, Sheng YY et al. }{ }^{19}\end{array}$ & $\begin{array}{l}\text { The difference in medical costs between carbapenem-resistant } \\
\text { Acinetobacter baumannii and non-resistant groups: a case study } \\
\text { from a hospital in Zhejiang province, China }\end{array}$ & 2017 & $\begin{array}{l}\text { European Journal of Clinical } \\
\text { Microbiology \& Infectious } \\
\text { Diseases. }\end{array}$ & China \\
\hline $\begin{array}{l}\text { Meng } X \text {, Liu S, Duan J, Huang X, } \\
\text { Zhou P, Xiong X et al. }\end{array}$ & $\begin{array}{l}\text { Risk factors and medical costs for healthcare-associated } \\
\text { carbapenem-resistant Escherichia coli infection among } \\
\text { hospitalized patients in a Chinese teaching hospital }\end{array}$ & 2017 & BMC Infectious Diseases & China \\
\hline $\begin{array}{l}\text { Johnston KJ, Thorpe KE, Jacob JT, } \\
\text { Murphy DJ. }\end{array}$ & $\begin{array}{l}\text { The incremental cost of infections associated with } \\
\text { multidrugresistant organisms in the inpatient hospital setting }-\mathrm{A} \\
\text { national estimate }\end{array}$ & 2019 & Health Services Research & Estados Unidos \\
\hline $\begin{array}{l}\text { Nguyen } \mathrm{HQ} \text {, Nguyen NT, Hughes } \\
\mathrm{CM}, \mathrm{O}^{\prime} \text { Neill C. }\end{array}$ & $\begin{array}{l}\text { Trends and impact of antimicrobial resistance on older inpatients } \\
\text { with urinary tract infections (UTIs): A national retrospective } \\
\text { observational study }\end{array}$ & 2019 & PLOS ONE & Estados Unidos \\
\hline $\begin{array}{l}\text { Kousoli E, Zarkotou O, Polimeri K, } \\
\text { Themeli-Digalaki K, Pournaras S. }\end{array}$ & $\begin{array}{l}\text { Impact of bloodstream infections caused by carbapenem- } \\
\text { resistant Gram-negative pathogens on ICU costs, mortality and } \\
\text { length of stay }\end{array}$ & 2019 & Infection Prevention in Practice & Grécia \\
\hline $\begin{array}{l}\text { Axente C, Licker M, Moldovan R, } \\
\text { Hogea E, Muntean D, Horhat F et } \\
\text { al. }^{24}\end{array}$ & $\begin{array}{l}\text { Antimicrobial consumption, costs and resistance patterns: a two } \\
\text { year prospective study in a Romanian intensive care unit }\end{array}$ & 2017 & BMC Infectious Diseases & Romênia \\
\hline $\begin{array}{l}\text { Naylor NR, Pouwels KB, Hope R, } \\
\text { Green N, Henderson KL, Knight } \\
\text { GM et al. }{ }^{25}\end{array}$ & $\begin{array}{l}\text { The health and cost burden of antibiotic resistant and } \\
\text { susceptible Escherichia coli bacteraemia in the English hospital } \\
\text { setting: A national retrospective cohort study }\end{array}$ & 2019 & PLOS ONE & Inglaterra \\
\hline $\begin{array}{l}\text { Vargas-Alzate } \quad \text { CA, Higuita- } \\
\text { Gutiérrez LF, Jiménez-Quiceno } \\
\text { JN. }{ }^{26}\end{array}$ & $\begin{array}{l}\text { Costos médicos directos de las infecciones del tracto urinario por } \\
\text { bacilos Gram negativos resistentes a betalactámicos en un } \\
\text { hospital de alta complejidad de Medellín, Colombia }\end{array}$ & 2018 & Biomédica (Bogotá) & Colômbia \\
\hline $\begin{array}{l}\text { Judd WR, Ratliff PD, Hickson RP, } \\
\text { Stephens DM, Kennedy CA. }{ }^{27}\end{array}$ & $\begin{array}{l}\text { Clinical and economic impact of meropenem resistance in } \\
\text { Pseudomonas aeruginosa-infected patients }\end{array}$ & 2016 & $\begin{array}{l}\text { American Journal of Infection } \\
\text { Control }\end{array}$ & Estados Unidos \\
\hline $\begin{array}{l}\text { Riu M, Chiarello P, Terradas R, Sala } \\
\text { M, Garcia-Alzorriz E, Castells X et } \\
\text { al. }^{28}\end{array}$ & $\begin{array}{l}\text { Incremental cost of nosocomial bacteremia according to the } \\
\text { focus of infection and antibiotic sensitivity of the causative } \\
\text { microorganism in a university hospital }\end{array}$ & 2017 & Medicine (Baltimore) & Espanha \\
\hline
\end{tabular}
al. $^{28}$ microorganism in a university hospital

Colloq Vitae 2021 mai-ago; 13(2): 25-39.

Artigo Open Access sob uma licença CC BY-NC-ND (http://creativecommons.org/licenses/by-nc-nd/4.0/). 
Tabela 3. Principais dados extraídos dos estudos selecionados, com autores, tipo de estudo, amostra, principais antimicrobianos e ônus

\begin{tabular}{|c|c|c|c|c|}
\hline Autores & Tipo de estudo & Amostra & Principais antimicrobianos & Ônus gerado \\
\hline $\begin{array}{l}\text { Phodha T, Riewpaiboon A, } \\
\text { Malathum A, Coyte PC }\end{array}$ & $\begin{array}{ll}\text { A, } & \text { Coorte } \\
& \text { retrospectivo }\end{array}$ & $\begin{array}{l}201 \text { pacientes } \\
\text { (151 RAM/ } 46 \text { controles) }\end{array}$ & Não especificado & $\begin{array}{l}\text { - Custos totais médios por caso: } \\
\text { RAM: \$ } 28.000 \\
\text { Não RAM: } \$ 24.000 \\
\text { - Custos médios (antibióticos): } \\
\text { AMR: \$ } 2.400 \\
\text { Não AMR: } \$ 2.100\end{array}$ \\
\hline $\begin{array}{l}\text { Zhen X, Chen Y, Hu X, Dong } \\
\text { P, Gu S, Sheng YY et al. }{ }^{19}\end{array}$ & $\begin{array}{l}\text { Coorte } \\
\text { retrospectivo }\end{array}$ & $\begin{array}{l}2.980 \text { pacientes } \\
\text { ( } 2.126 \text { casos/ } 854 \text { controles) }\end{array}$ & $\begin{array}{l}\text { - Beta-lactamases: } 1495 \\
\text { (50,2\%) } \\
\text { - Carbapenêmicos: } 1489 \\
\text { (50,0\%) } \\
\text { - Glicopeptídeos: } 660 \\
(22,1 \%)\end{array}$ & $\begin{array}{l}\text { - A. baumannii resistente a carbapenem: } \\
\text { Custo médico total: } \$ 30.575,14 \\
\text { Custo de medicação: } \$ 11.711,80 \\
\text { Custo de antibióticos: } \$ 3.047,32 \\
\text { Custo hospitalar: } \$ 10.364,47 \\
\text { - A. baumannii não resistente: } \\
\text { Custo médico total: } \$ 19.783,49 \\
\text { Custo de medicação: } \$ 7.226,18 \\
\text { Custo de antibióticos: } \$ 1.691,86 \\
\text { Custo hospitalar: } \$ 6.125,04\end{array}$ \\
\hline $\begin{array}{l}\text { Meng X, Liu S, Duan J, } \\
\text { Huang X, Zhou P, Xiong X et } \\
\text { al. }^{20}\end{array}$ & $\begin{array}{l}\text { Observacional } \\
\text { retrospectivo }\end{array}$ & $\begin{array}{l}147 \text { pacientes } \\
\text { (49 casos/ } 98 \text { controles) }\end{array}$ & $\begin{array}{l}\text { Cefalosporina: } \\
\text { - CREC: } 36(73 \%) \\
\text { - CSEC: } 52(53 \%) \\
\text { - S.I.: } 28(29 \%) \\
\text { Carbapênemicos: } \\
\text { - CREC: } 19(38 \%) \\
\text { - CSEC: } 19(19 \%) \\
\text { - S.I.: } 4(4 \%)\end{array}$ & $\begin{array}{l}\text { Caso (resistente): } \\
\text {-Totais: \$ } 11.590 \\
\text {-Medicamento: } \$ 6.255 \\
\text {-Anti-infeccioso: \$1.308 } \\
\text { Controle 1(suscetível): } \\
\text {-Totais: } \$ 9.413,1 \\
\text {-Medicamento: } \$ 4.267,6 \\
\text {-Anti-infeccioso: } \$ 708,06 \\
\text { Controle } 2 \text { (sem infecção): } \\
\text {-Totais: } \$ 2.578,2 \\
\text {-Medicamento: } \$ 963,74 \\
\text {-Anti-infeccioso: } \$ 17,92 \\
\text { - Mortalidade: } \\
\text { Caso (resistente): } 12 \% \text { (n=6/49) } \\
\text { Controle } 1 \text { (suscetível): } 1 \%(n=1 / 96) \\
\text { Controle } 2 \text { (sem infecção): } 1 \%(n=1 / 96)\end{array}$ \\
\hline Johnston KJ, Thorpe KE, & Observacional & 6.385 .258 internações & Não especificado & - Custo adicional por internação: \\
\hline
\end{tabular}

Colloq Vitae 2021 mai-ago; 13(2): 25-39.

Artigo Open Access sob uma licença CC BY-NC-ND (http://creativecommons.org/licenses/by-nc-nd/4.0/). 
Jacob JT, Mur-phy DJ. ${ }^{21}$

retrospectivo
MRSA: $\$ 1.718(1.609-1.826)$

C. difficile: \$ 4.617 (4.407-4.827)

Outra MDRO: \$ 2.302 (2.044-2.560)

Multiplos MDROs: \$ 3.570 (3.019-4.122)

- Tempo adicional de internação:

MRSA: 1,04 (0,99-1,09)

Clostridium difficile: $2,62(2,52-2,73)$

Outra MDRO: 1,31 (1,20-1,42)

Multiplas infecções MDRO: 1,53 (1,29-

1,76)

Nguyen $\mathrm{HQ}$, Nguyen NT,

Hughes $\mathrm{CM}$, O'Neill C. ${ }^{22}$

Observacional

546.305 internações

(43.408 casos/ 521.947

Não especificado

controles)

- Custos hospitalares:

Não-RA: \$ 5.641 (3.901-8.413)

RA: $\$ 7.383(4.924-11.414)$

BR: $\$ 7.021(4.833-10.478)$

MRSA: $\$ 8.038$ (5.403-12.548)

MR: \$ 6.757 (4.457-10.415)

QR: \$ 6.194 (4.286-8.947)

- Período de internação (dias):

Não-RA: 3 (2-5)

RA: 5 (3-7)

BR: $4(3-6)$

MRSA: $5(4-8)$

MR: 5 (3-7)

QR: 4 (3-5)

Kousoli E, Zarkotou O,

Polimeri K, Themeli-

Observacional

419 pacientes

Não especificado

- Custo total:

Digalaki K, Pournaras $\mathrm{S}^{23}$

\begin{abstract}
retrospectivo
(142 casos / 277 controles)
\end{abstract}

CRGN BSIs: \$22.883,6

Não CRGN BSIs: \$ 9.564,59

- Tempo de internação:

CRGN BSIs:30,0 dias

Não CRGN BSIs: 12,0 dias

- Mortalidade: $33,7 \%$ (49,3/25,6\% casos/controles).

\begin{tabular}{lllll} 
Axente C, Licker M, & Coorte & 1.596 pacientes divididos em: & - Cefalosporinas (I - V & - Custos: \\
Moldovan R, Ho-gea E, & prospectivo & SI - pacientes com doenças & geração): 264,19 & Profilático: \$ 10,54 / doente-dia \\
Muntean D, Horhat F et & & não infecciosas que receberam & - Carbapenêmicos: 244,59 & IAC: \$ 37,16 / doente-dia \\
\hline
\end{tabular}

Colloq Vitae 2021 mai-ago; 13(2): 25-39.

Artigo Open Access sob uma licença CC BY-NC-ND (http://creativecommons.org/licenses/by-nc-nd/4.0/). 


\begin{tabular}{|c|c|c|c|c|}
\hline al. $^{24}$ & & $\begin{array}{l}\text { antibioticoterapia profilática; } \\
\text { SII - pacientes com IAC ou } \\
\text { complicações infecciosas de } \\
\text { doenças crônicas. } \\
\text { SIII - pacientes que } \\
\text { desenvolveram HAI } 48 \text { horas } \\
\text { ou mais após a admissão na } \\
\text { UTI; SIV - todos os casos de IAC } \\
\text { no momento da admissão, } \\
\text { complicados por IRAS }\end{array}$ & $\begin{array}{l}\text { - Glicopeptídeos: } \\
\text { 132,94 } \\
\text { - Polimixinas: } \\
\text { 115,47 } \\
\text { - Penicilinas: } 109,61 \\
\text { (DDD } 1000 \text { pacientes/dia) }\end{array}$ & $\begin{array}{l}\text { HAl: \$ 41,71 / doente-dia } \\
\text { HA: \$ 56,62 / doente-dia } \\
\text { - Tempo de internação: } 6,70 \text { dias para } \\
\text { pacientes com infecções adquiridas na } \\
\text { comunidade vs } 16,06 \text { / 14,08 dias para } \\
\text { aqueles com infecções adquiridas no } \\
\text { hospital } \\
\text { - Mortalidade: } 45,16 \% \text { (com variações de } \\
28,12 \% \text { no SI, a 52,03\% no SII, 59,27\% no } \\
\text { SIII e } 65,21 \% \text { no SIV). }\end{array}$ \\
\hline $\begin{array}{l}\text { Vargas-Alzate CA, Higuita- } \\
\text { Gutiérrez LF, Jiménez- } \\
\text { Quiceno JN. }{ }^{26}\end{array}$ & Coorte & $\begin{array}{l}141 \text { pacientes } \\
\text { (86 casos/ } 55 \text { controles) }\end{array}$ & $\begin{array}{l}\text { - Resistente a } \\
\text { cefalosporinas: } \\
\text { meropenem intravenoso } \\
\text { - Resistente a } \\
\text { carbapenêmicos: } \\
\text { fosfomicina (um caso com } \\
\text { fosfomicina mais } \\
\text { cefepima) e meropenem } \\
\text { (dois casos com } \\
\text { meropenem mais } \\
\text { polimixina B e outro com } \\
\text { meropenem mais } \\
\text { fosfomicina), ambos por }\end{array}$ & $\begin{array}{l}\text { - Custo médio total da internação: } \\
\text { Sensível a beta-lactâmicos: \$ } 892 \text { (IQR = } \\
601-1.577) \\
\text { Resistente às cefalosporinas: \$1.236 (IQR } \\
\text { = 909-1.854) } \\
\text { Resistente a carbapenêmicos: \$ } 1.473 \\
\text { (IQR: 916-2.944) } \\
\text { - Período médio de internação: } \\
\text { Sensível a beta-lactâmicos: } 8 \text { (IQR = 5-11) } \\
\text { Resistente às cefalosporinas: } 10 \text { (IQR = 7- } \\
\text { 12) } \\
\text { Resistente a carbapenêmicos: } 10 \text { (IQR = } \\
6,5-14)\end{array}$ \\
\hline
\end{tabular}

Colloq Vitae 2021 mai-ago; 13(2): 25-39.

Artigo Open Access sob uma licença CC BY-NC-ND (http://creativecommons.org/licenses/by-nc-nd/4.0/) 


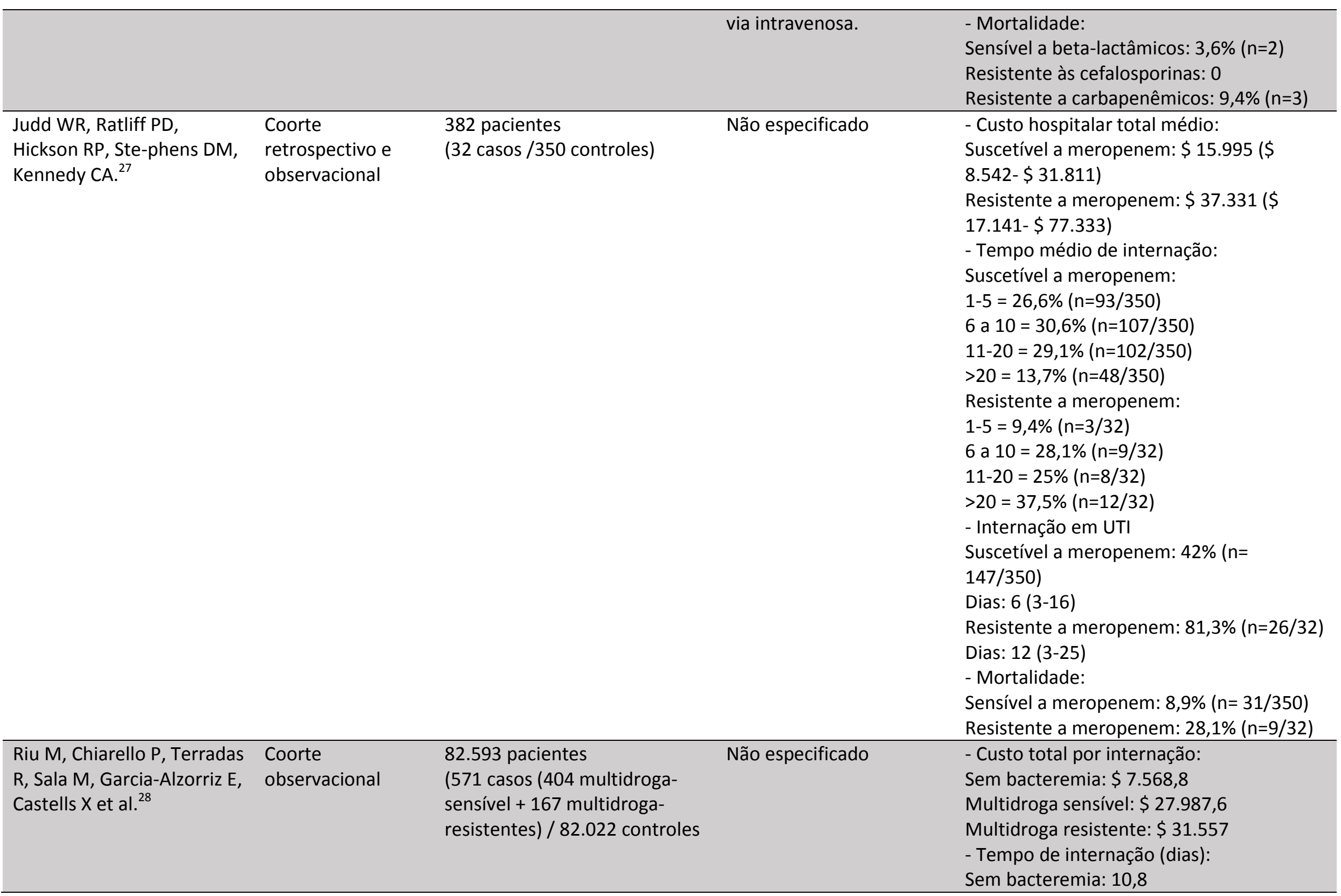

Colloq Vitae 2021 mai-ago; 13(2): 25-39.

Artigo Open Access sob uma licença CC BY-NC-ND (http://creativecommons.org/licenses/by-nc-nd/4.0/). 


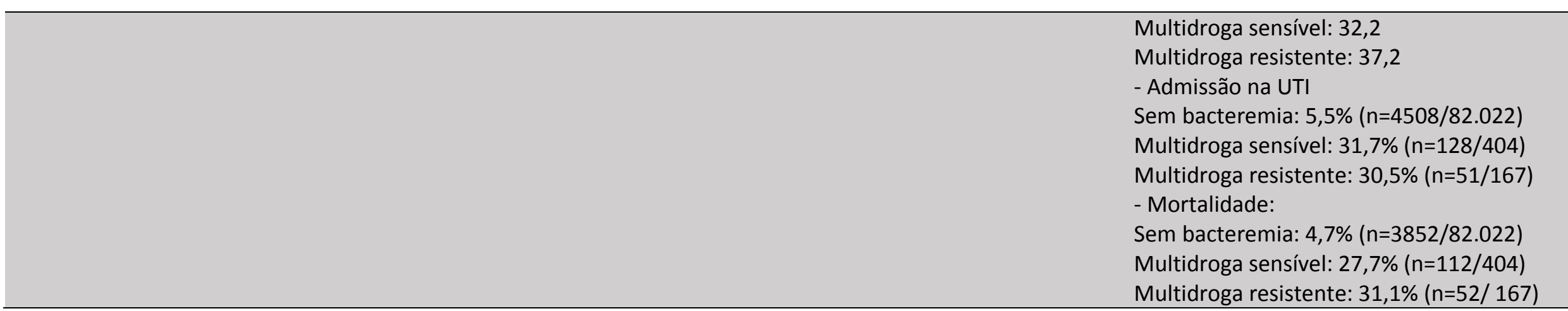

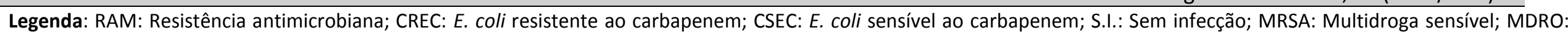

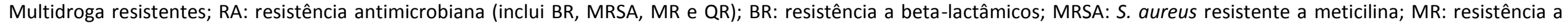

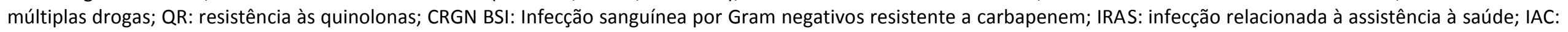
Infecção adquirida na comunidade; HAl: Infecção hospitalar; HA: Isolados adquiridos em hospitais.

Colloq Vitae 2021 mai-ago; 13(2): 25-39.

Artigo Open Access sob uma licença CC BY-NC-ND (http://creativecommons.org/licenses/by-nc-nd/4.0/). 


\section{DISCUSSÃO}

Os resultados dos estudos incluídos na presente revisão sistemática apontam diferenças consideráveis em todos os aspectos observados ao se comparar grupos de pacientes acometidos por bactérias resistentes e não resistentes, limitadas não apenas na perspectiva econômica, mas também no período médio de hospitalização, necessidade de internação em leito de UTI e índice de mortalidade.

Visualizando os gastos médios por internação, todos os onze estudos relataram aumento considerável de custos, variando de $344^{26}$ a 23.989 dólares ${ }^{28}$ adicionais por paciente/internação em casos de infecções causadas por bactérias resistentes.

Quanto aos custos dos antimicrobianos, apenas três trabalhos determinaram especificamente os gastos com o medicamento: um estudo de coorte retrospectivo ${ }^{19}$ feito com 2.980 pacientes, um caso-controle retrospectivo ${ }^{20}$ realizado com 147 pacientes, ambos sendo realizados na China em 2017, e um estudo de coorte retrospectivo ${ }^{18}$ feito com 201 pacientes na Tailândia em 2019, com o primeiro analisando o impacto da Acinetobacter baumannii resistente a carbapenêmicos, enquanto o segundo compara os custos da Escherichia coli resistentes a carbapenêmicos e o último observa os excessos de custos provocados por infecções nosocomiais multirresistentes.

As informações levantadas por Zhen X e colaboradores $^{19}$ apontam que os gastos médios específicos com antimicrobianos chegam a 3.047 dólares no grupo de pacientes acometidos pela $A$. baumannii resistente a carbapenem, já no grupo não resistente, esse valor vai a 1.691 dólares. 0 estudo feito por Meng $X$ e colaboradores ${ }^{20}$ mostra uma média de gastos de 1.308 dólares para pacientes com $E$. coli resistentes a carbapenem, 708 dólares para pacientes suscetíveis ao medicamento e 17 dólares para pacientes sem infecção alguma. Essa discrepância de valores possivelmente se deve a uma diferença de gravidade e complicações entres as infecções causadas pelas bactérias $A$. baumannii e E.coli. Por fim, o estudo realizado por Phodha T e colaboradores ${ }^{18}$ indica que os custos médios com antibióticos no grupo multirresistente chegaram a 2.400 dólares, enquanto que no grupo controle, os valores não passam de $\mathbf{2 . 1 0 0}$ dólares.

Observa-se o grande consumo de medicamentos da classe dos $\beta$-lactâmicos, sendo essa de longe a mais utilizada, possivelmente devido à sua baixa toxidade e amplo espectro de ação, além de ser considerado tratamento de primeira linha em diversos casos como infeç̧ões de pele e tecidos moles, trato respiratório, osteoarticular, trato urinário, sistema nervoso central, endocardite bacteriana, sepse e neutropenia febril ${ }^{17}$.

O estudo de coorte prospectivo ${ }^{24}$ realizado com 1.596 pacientes divididos em quatro subgrupos em uma unidade de terapia intensiva na Romênia em 2017 mostra que dos três antimicrobianos mais consumidos no local (Cefalosporinas ( $\mathrm{-V}$ geração, Carbapenêmicos e Glicopeptídeos), os dois primeiros pertencem a classe dos $\beta$-lactâmicos. Nesse mesmo estudo o consumo, de antibióticos atingiu o valor de 1080,38 DDD / 1000 pacientes-dia, tendo a seguinte distribuição: $14,19 \%$ para profilaxia antibiótica, 15,83\% para pacientes com IAC (infecções adquiridas na comunidade), 63,64\% para pacientes com HAl (infecções adquiridas no hospital) e 6,33\% para aqueles com infecções adquiridas associada a IRAS (infecções relacionadas à assistência à saúde). Outros três trabalhos que também especificam o consumo de antimicrobianos em suas populações ${ }^{19,20,26}$ corroboram os dados do estudo previamente citado.

Pode-se observar também o aumento da taxa de mortalidade decorrente de infecções bacterianas resistentes, isso fica claro no estudo de coorte observacional ${ }^{28}$ realizado em 2017 com 82.593 pacientes na Espanha em um hospital universitário, cujo índice de mortalidade chega a apenas $4,7 \% \mathrm{em}$ pacientes sem bacteremia $(n=3.852 / 82.022)$, enquanto que em pacientes com infecção multirresistentes esse número passa de $31 \%(n=52 / 167)$.

Nota-se ainda que dos oito estudos selecionados $^{21-28}$ que trazem informações a respeito do tempo médio de internação de pacientes acometidos por uma infecção bacteriana resistente, todos apontam para um aumento desse período de hospitalização, que segundo o estudo de coorte prospectivo ${ }^{24}$ realizado na Romênia, pode chegar a uma diferença de aproximadamente 7 dias para pacientes com infecções adquiridas na comunidade para até 16 dias em pacientes com infecções hospitalares, o que gera complicações tanto para hospitais e sistemas de saúde, com o "bloqueio de leitos" prejudicando a rotatividade de pacientes e diminuindo suas capacidades 
operacionais, como também para o próprio paciente, que com o isolamento e internação prolongada, pode acabar sofrendo efeitos sociais e psicológicos negativos como ansiedade, depressão, baixa autoestima e sensação de controle reduzida ${ }^{29}$.

Apenas dois estudos especificaram em seus desfechos a demanda por transferência para leitos de UTI por complicações provocados pela RAM, um estudo de coorte ${ }^{27}$ norte americano feito em 2016 com 382 pacientes, e outro também de coorte realizado com 82.593 pacientes em 2017 na Espanha ${ }^{28}$.

Judd WR e colaboradores ${ }^{27}$ fizeram uma comparação entre pacientes infectados com a Pseudomonas aeruginosa suscetíveis e resistentes ao antimicrobiano meropenem, onde $42 \%(n=147 / 350)$ do grupo de não resistentes necessitou de atendimento em unidade de terapia intensiva por um período médio de 6 dias, enquanto que a porcentagem de pacientes do grupo resistente que necessitaram desse tipo de atendimento quase dobrou, chegando a $81,3 \%$ ( $n=26 / 32$ ), o período médio de internação em leito de UTI também dobrou, tendo chegado a 12 dias, e em alguns casos até 25 dias. Já Riu M e colaboradores $^{28}$ se propuseram a comparar grupos de pacientes com bacteremias nosocomiais sensível e resistente, e sem bacteremia alguma, ficando a porcentagem de admissões em UTI, respectivamente, em: $31,7 \%$ $(n=128 / 404), 30,5 \%(n=51 / 167)$ e apenas $5,5 \%$ $(n=4508 / 82.022)$ em pacientes sem sinais de bacteremia.

Fica evidente que ainda há poucos trabalhos realizados no Brasil que tratem dos custos monetários gerados pela RAM, não tendo nenhum estudo encontrado atendido aos critérios de seleção da presente revisão. Porém, é valido citar um estudo feito por Barros GR e colaboradores $^{30}$, que apenas não foi incluído nesta revisão sistemática por não comparar os dados encontrados na população de pacientes resistentes com grupos não resistentes ou sem infecção. Esse estudo do tipo descritivo, retrospectivo e transversal contendo 90 pacientes diagnosticados com resistência a múltiplos antimicrobianos foi realizado em um hospital público de alta complexidade no Agreste Pernambucano, entre fevereiro e junho de 2017. Foi observado que Meropenem foi o antibiótico mais administrado no tratamento de bactérias Gram negativas multirresistentes. Para pacientes Gram positivos, Vancomicina, Piperacilina com
Tazobactam, Ampicilina com Sulbactam e Cefepime foram tratamentos mais utilizados, com custo anual da antibioticoterapia, em reais, de $\mathrm{R} \$$ 83.298,83 (aproximadamente 15.520 dólares convertidos). Cerca de 48,9\% ( $n=44)$ dos pacientes com infecções multirresistentes morreram. Estes números são compatíveis com os dados apresentados pelos demais estudos selecionados.

Um ponto forte da presente revisão sistemática foi a inclusão de estudos com grandes tamanhos de amostragem, o que traz mais confiabilidade aos dados obtidos. Quanto as suas limitações, é valido citar que os estudos foram selecionados por apenas um pesquisador, o que pode ter aumentado o risco de viés. Também deve ser mencionado que não foram feitas buscas na literatura cinzenta (teses, relatórios institucionais ou governamentais e publicações periódicas de baixa visibilidade e distribuição), diminuindo o alcance deste estudo.

Ao observar os dados retirados destes estudos é possivel perceber que o aumento de custos está relacionado principalmente com o prolongamento do período de internação devido às complicações clínicas motivadas pela resistência antimicrobiana, gerando custos diretos (com o aumento do valor do tratamento em si) e indiretos (prejudicando a rotatividade de pacientes e diminuindo as capacidades operacionais do hospital). A necessidade da utilização de antimicrobianos mais potentes, em conjunto com outros medicamentos e em maiores quantidades, elevações na taxa de mortalidade e necessidade de internação em leitos de UTI também foram observadas.

É perceptível a necessidade de mais estudos, tanto ao nível global quanto nacional, com maiores níveis de evidência e que avaliem as perdas financeiras motivadas pela resistência microbiana, como estudos de coorte do tipo prospectivo (nível 2B de evidência ${ }^{31}$ ).

\section{AGRADECIMENTOS E CONFLITO DE INTERESSES}

Agradeço a bibliotecária Jakeline Margaret de Queiroz Ortega por realizar a montagem das estratégias de buscas utilizadas nesta revisão sistemática.

Os autores declaram não haver qualquer potencial conflito de interesse que possa interferir na imparcialidade deste trabalho científico. 


\section{REFERÊNCIAS}

1. Silva P. Farmacologia. 8. ed. Rio de Janeiro: Guanabara Koogan; 2010.

2. Sáez-Llorens $X$, Wong $M$, Castaño $E$, Suman $O$, Morös D, Atencio I. Impact of an antibiotic restriction policy on hospital expenditures and bacterial susceptibilities: a lesson from a pediatric institution in a developing country. PIDJ. 2000; 19(3):200-206. DOI: https://dx.doi.org/10.1097/00006454200003000-00005

3. Apolônio A, Machado A. Microbiologia Bucal e Aplicada. 1. ed. Rio de Janeiro: Guanabara Koogan; 2018.

4. Finland M. Changing Patterns of Resistance of Certain Common Pathogenic Bacteria to Antimicrobial Agents. NEJM. 1955; 252(14):570-580.

5. Abraham E, Chain E. An Enzyme from Bacteria able to Destroy Penicillin. Nature. 1940; 146(3713):837-837.

6. Gaynes R. The Discovery of PenicillinNew Insights After More Than 75 Years of Clinical Use. Emerg. Infect. Dis. 2017; 23(5):849-853. DOI: https://dx.doi.org/10.3201/eid2305.161556

7. What is antimicrobial resistance? World Health Organization. 2017 [cited 2019 may 15]. Disponível em: https://www.who.int/features/qa/75/en/

8. Munita J, Arias C. Mechanisms of Antibiotic Resistance. Microbiol. Spectr. 2016; 4 (2).

DOI: https://dx.doi.org/10.1128/microbiolspec.VMBF$\underline{0016-2015}$

9. Santos N. A resistência bacteriana no contexto da infecção hospitalar. Texto e Contexto Enferm. 2004; 13(spe):64-70. DOI: https://dx.doi.org/10.1590/S010407072004000500007

10. O'Neill J. The Review on Antimicrobial Resistance. Tackling Drug-Resistant Infections Globally: Final Report and Recommendations. London:AMR; 2016.
11. World Health Organization. Global action plan on antimicrobial resistance [Internet]. 2015 [cited 2019 may 15]. Available from: https://www.who.int/antimicrobialresistance/publications/global-action-plan/en/

12. World Health Organization. Ten threats to global health in 2019 [Internet]. 2019 [cited 2019 may 15]. Available from: https://www.who.int/emergencies/ten-threatsto-global-health-in-2019

13. McKenna M. Antibiotic resistance: The last resort. Nature. 2013; 499(7459):394-396. DOI: https://dx.doi.org/10.1038/499394a

14. OECD. Stemming the Superbug Tide: Just A Few Dollars More - OECD Health Policy Studies. Paris: OECD Publishing; 2018.

15. Moher D, Liberati A, Tetzlaff J, Altman DG, The PRISMA Group. Preferred Reporting Items for Systematic Reviews and Meta-Analyses: The PRISMA Statement. PLoS Med. 2019 Jul. 21;6(7) DOI: https://dx.doi.org/10.1371/journal.pmed.100009 $\underline{7}$

16. Costa SC, Mattos PC, Cuce NM. A estratégia PICO para a construção da pergunta de pesquisa e busca de evidências. Rev. Lat. Am. Enfermagem. 2007; 15(3):508-511. DOI: https://dx.doi.org/10.1590/S010411692007000300023

17. Suárez C, Gudiol F. Antibióticos betalactámicos. Enferme. Infecc. Microbiol. Clin. 2009; 27:116-129. DOI: https://dx.doi.org/10.1016/j.eimc.2008.12.001

18. Phodha T, Riewpaiboon A, Malathum A, Coyte PC. Excess annual economic burdens from nosocomial infections caused by multi-drug resistant bacteria in Thailand. Expert Rev. Pharmacoeconomics Outcomes Res. 2018 Oct 19;19(3):305-312.

DOI: https://dx.doi.org/10.1080/14737167.2019.1537 $\underline{123}$

19. Zhen X, Chen Y, Hu X, Dong P, Gu S, Sheng $Y Y$ et al. The difference in medical costs between carbapenem-resistant Acinetobacter baumannii and non-resistant groups: a case study from a 
hospital in Zhejiang province, China. Eur. J. Clin. Microbiol. Infect. Dis. 2017 Aug 23;36:1989-1994. DOI: $\quad$ https://dx.doi.org/10.1007/s10096-0173088-3

20. Meng X, Liu S, Duan J, Huang X, Zhou P, Xiong $X$ et al. Risk factors and medical costs for healthcare-associated carbapenem-resistant Escherichia coli infection among hospitalized patients in a Chinese teaching hospital. BMC Infect. Dis. 2017 Jan $17 . \quad$ DOI: https://dx.doi.org/10.1186/s12879-016-2176-9

21. Johnston KJ, Thorpe KE, Jacob JT, Murphy DJ. The incremental cost of infections associated with multidrug-resistant organisms in the inpatient hospital setting-A national estimate. Health Serv. Res. 2019 Mar 12;54(4):782-792. DOI: $\quad$ https://dx.doi.org/10.1111/1475-

\section{$\underline{6773.13135}$}

22. Nguyen $H Q$, Nguyen NT, Hughes $C M$, O'Neill C. Trends and impact of antimicrobial resistance on older inpatients with urinary tract infections (UTIs): A national retrospective observational study. PLOS ONE. 2019 Oct 03;14(10). DOI: https://dx.doi.org/10.1371/journal.pone.022340 $\underline{9}$

23. Kousoli E, Zarkotou O, Polimeri K, Themeli-Digalaki K, Pournaras S. Impact of bloodstream infections caused by carbapenemresistant Gram-negative pathogens on ICU costs, mortality and length of stay. IPIP. 2019 June;1(2) DOI:

\section{https://dx.doi.org/10.1016/j.infpip.2019.100020}

24. Axente $\mathrm{C}$, Licker M, Moldovan R, Hogea E, Muntean D, Horhat $F$ et al. Antimicrobial consumption, costs and resistance patterns: a two year prospective study in a Romanian intensive care unit. BMC Infect. Dis. 2017 May 22;17(358).

DOI: https://dx.doi.org/10.1186/s12879-017-2440-7

25. Naylor NR, Pouwels KB, Hope R, Green N, Henderson KL, Knight GM et al. The health and cost burden of antibiotic resistant and susceptible Escherichia coli bacteraemia in the English hospital setting: A national retrospective cohort study. PLOS ONE. 2019 Sep 10;14(9). DOI: https://dx.doi.org/10.1371/journal.pone.022194 $\underline{4}$

26. Vargas-Alzate CA, Higuita-Gutiérrez LF, Jiménez-Quiceno JN. Costos médicos directos de las infecciones del tracto urinario por bacilos Gram negativos resistentes a betalactámicos en un hospital de alta complejidad de Medellín, Colombia / Direct medical costs of urinary tract infections by Gram-negative bacilli resistant to beta-lactams in a tertiary care hospital in Medellín, Colombia. Biomédica. 2019 May; 39:3549.

DOI:

https://dx.doi.org/10.7705/biomedica.v39i1.3981

27. Judd WR, Ratliff PD, Hickson RP, Stephens DM, Kennedy CA. Clinical and economic impact of meropenem resistance in Pseudomonas aeruginosa-infected patients. Am. J. Infect. Control. 2016 Nov 01;44(11):1275-1279. DOI:

https://dx.doi.org/10.1016/j.ajic.2016.04.218

28. Riu $M$, Chiarello $P$, Terradas $R$, Sala $M$, Garcia-Alzorriz E, Castells $X$ et al. Incremental cost of nosocomial bacteremia according to the focus of infection and antibiotic sensitivity of the causative microorganism in a university hospital. Medicine (Baltimore). 2017 Apr 28;96(17). DOI: https://dx.doi.org/10.1097/md.00000000000066 $\underline{45}$

29. Gammon J. Analysis of the stressful effects of hospitalisation and source isolation on coping and psychological constructs. Int J Nurs Pract. 1998 Jun;4(2):84-96. DOI: https://dx.doi.org/0.1046/j.1440$\underline{172 x .1998 .00084 . x}$

30. Barros GR, Melo CE, Oliveira MI, Germinio $\mathrm{JE}$, Santos RJ, Oliveira SR. Impacto financeiro da antibioticoterapia na resistência a múltiplas drogas bacterianas em um hospital de emergência em Pernambuco, Brasil. Arq. Pharmaceutica. 2020 Jul. 20;61(2):121-126. DOI: https://dx.doi.org/10.30827/ars.v61i2.115337

31. Medeiros LR, Stein A. Níveis de evidência e graus de recomendação da medicina baseada em evidências. Rev. AMRIGS. 2002;46(1,2):43-6. 